\title{
Construction of a Self-Purification \\ and Self-Assembly Coenzyme Regeneration System for the Synthesis of Chiral Drug Intermediates
}

Wei Jiang*, Wanru Zeng

College of Chemical Engineering, Huaqiao University, 668 Jimei Blvd., Xiamen,

Fujian, 361021, China

*Corresponding author:

Wei Jiang

E-mail: wjiang@hqu.edu.cn; tianya416@126.com

Tel.: $+86-05926162305$

Fax: +86-05926162305 
Supporting information

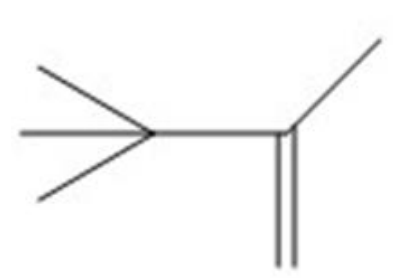

$\mathrm{O}$

TMA
$\mathrm{COOH}$

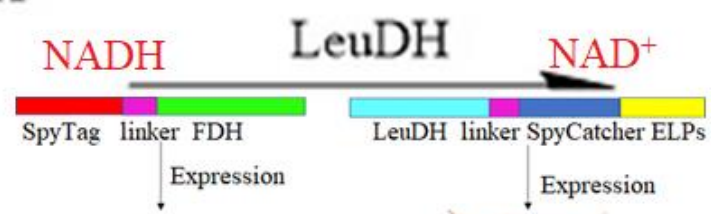

SpyCatcher

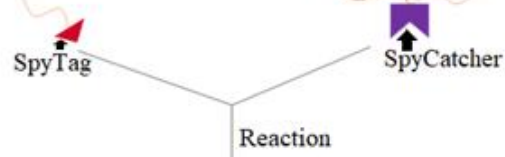

Reaction

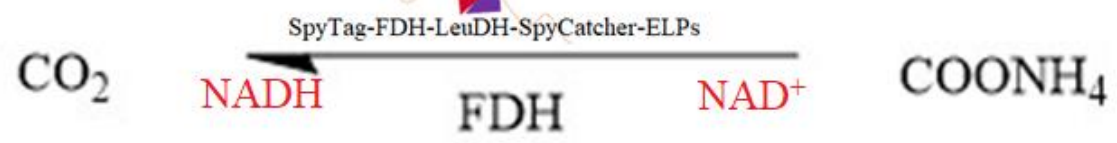

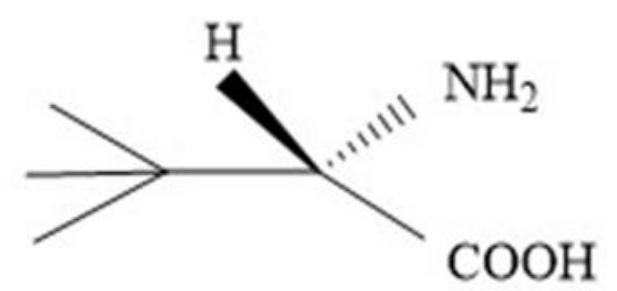

L-tert-Leucine

\section{L-tert-Leucine}

(n)



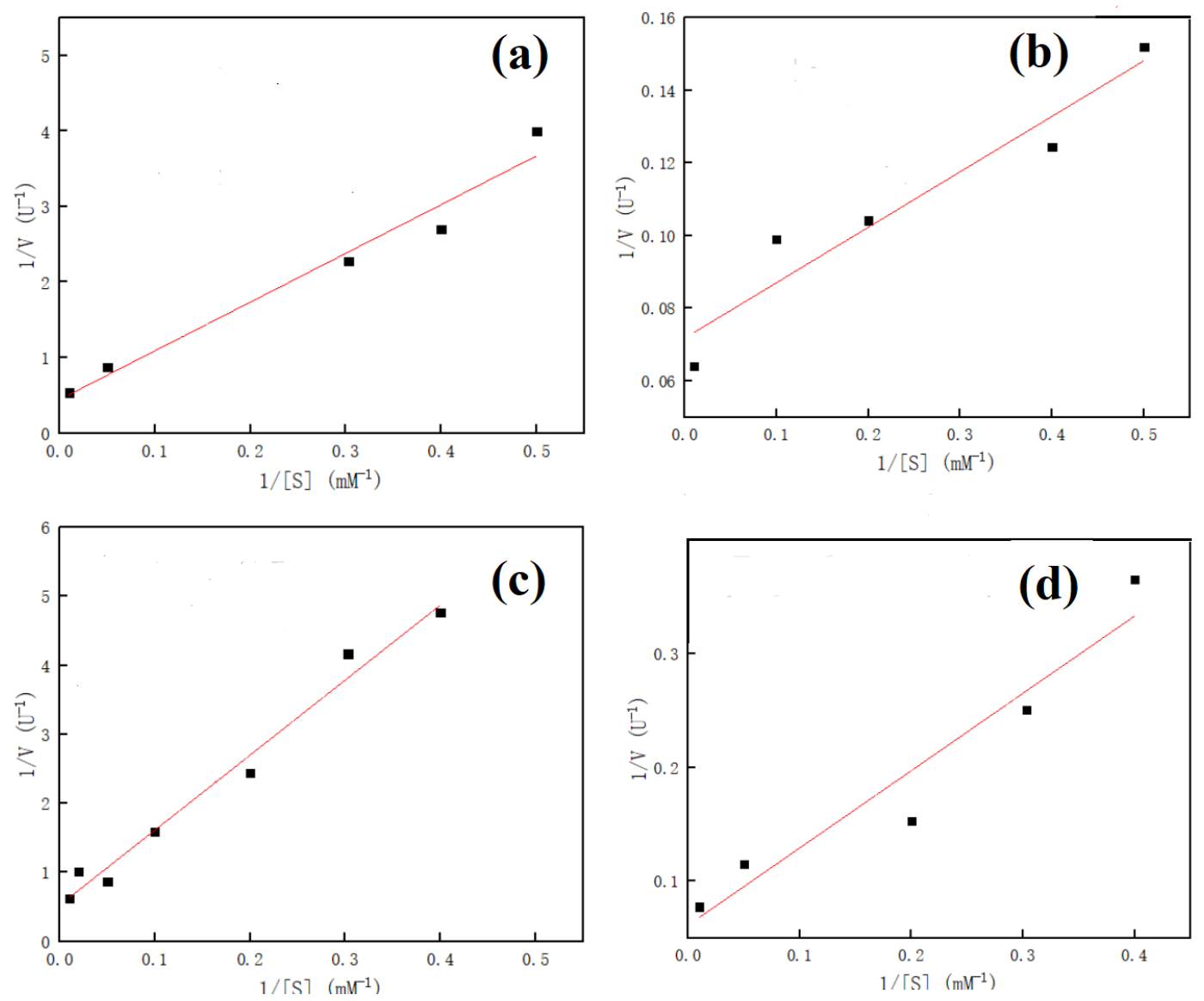

Figure S1 Double bottom figure of apparent kinetic parameters 
Note: (a) FR-LR (FR), 5 times dilution; (b) FR-LR (LR), 50 times dilution; (c) FR+LR (FR), 5 times dilution; (d) FR+LR (LR), 50 times dilution.

Full amino acid sequence of LeuDH-linker-SpyCatcher-ELPs (LR)

LeuDH-linker-SpyCatcher-ELPs

MTLEIFEYLEKYDYEQVVFCQDKESGLKAIIAIHDTTLGPALGGTRMWTYDSE EAAIEDALRLAKGMTYKNAAAGLNLGGAKTVIIGDPRKDKSEAMFRALGRYI QGLNGRYITAEDVGTTVDDMDIIHEETDFVTGISPSFGSSGNPSPVTAYGVYRG MKAAAKEAFGTDNLEGKVIAVQGVGNVAYHLCKHLHAEGAKLIVTDINKEA VQRAVEEFGATAVEPNEIYGVECDIYAPCALGATVNDETIPQLKAK VIAGSANN QLKEDRHGDIIHEMGIVYAPDYVINAGGVINVADELYGYNRERALKRVESIYD TIAKVIEISKRDGIATYVAADRLAEERIASLKNSRSTYLRNGHDIISRR GSGGSG GSGDSATHIKFSKRDEDGKELAGATMELRDSSGKTISTWISDGQVKDFYLYPG KYTFVETAAPDGYEVATAITFTVNEQGQVTVNGKATKGDAHIVPGVGVPGKG VPGVGVPGVGVPGVGVPGVGVPGVGVPGVGVPGVGVPGFGVPGVGVPGKG VPGVGVPGVGVPGVGVPGVGVPGVGVPGVGVPGVGVPGFG

Full amino acid sequence of SpyTag-linker-FDH (FR)

SpyTag-linker-FDH

AHIVMVDAYKPTKGSGGSGMKIVLVLYDAGKHAADEEKLYGSTENKLGIAN WLKDQGHELITTSDKEGETSELDKHIPDADIITTPFHPAYITKERLDKAKNLKL VVVAGVGSDHIDLDYINQTGKKISVLEVTGSNVVSVAEHVVMTMLVLVRNFV PAHEQIINHDWEVAAIAKDAYDIEGKTIATIGAGRIGYRVLERLLPFNPKELLYY DYQALPKEAEEKVGARRVENIEELVAQADIVTVNAPLHAGTKGLINKELLSKF KKGAWLVNTARGAICVAEDVAAALESGQLRGYGGDVWFPQPAPKDHPWRD 
MRNKYGAGNAMTPHYSGTTLDAQTRYAEGTKNILESFFTGKFDYRPQDIILL NGEYVTKAYGKHDKK. 